\title{
Cápsula endoscópica para el diagnóstico de metástasis de melanoma en intestino delgado, reporte de dos casos y revisón de la literatura
}

\author{
Hugo Cedron Cheng, ${ }^{1}$ Josué Aliaga Ramos ${ }^{2}$ \\ ${ }^{1}$ Gastroenterólogo Titular de la Clínica Anglo Americana. Unidad de Intestino Delgado. \\ ${ }^{2}$ Gastroenterologo-endoscopista. Servicio de Gastroenterologia, Clinica San Pablo-Surco. Facultad de Medicina, Universidad Peruana Cayetano Heredia. \\ Lima, Perú.
}

Acta Gastroenterol Latinoam 2020;50(1):50-56

Recibido: 20/05/2018 / Aceptado: 02/10/2018 / Publicado online: 23/03/2020 / bttps://doi.org/10.52787/llbj9802

\section{Resumen}

El melanoma es la neoplasia que produce con mayor frecuencia metástasis a nivel del tracto gastrointestinal. Se pueden encontrar metástasis en intestino delgado hasta en un 50-60\% de las necropsias de pacientes que fallecen por melanoma. Sin embargo, el diagnóstico de metástasis a nivel de intestino delgado in vivo se logra en menos del $10 \%$ de los pacientes. La cápsula endoscópica es considerada un excelente método diagnóstico para la evaluación del intestino delgado en este grupo de pacientes. Por tal motivo se debe considerar realizar este estudio para descartar compromiso a nivel de intestino delgado en todo paciente con melanoma, actual o previo, más aún si desarrolla hemorragia digestiva, anemia ferropénica, dolor abdominal y/o pérdida ponderal importante inexplicable. En el presente trabajo se presentan dos pacientes, previamente tratados por melanoma cutáneo, en aparente remisión, que desarrollaron anemia ferropénica, sin historia de pérdidas gastrointestinales evidentes, con exámenes endoscópicos convencionales negativos y con hallazgos compatibles en la cápsula endoscópica de metástasis intestinal.

Palabras claves. Cápsula endoscópica, melanoma maligno, intestino delgado, anemia ferropénica.

\section{Capsule Endoscopy in the Diagnosis of Melanoma Metastasis in the Small Bowel. Two Cases Report and Literature Review}

\section{Summary}

Malignant melanoma is the neoplasm that most frequently produces metastasis at the gastrointestinal tract. Metastatic lesions can be found in the small bowel in 50 to $60 \%$ of the necropsies of patients who die due to malignant melanoma; however, the diagnosis of metastasis in vivo is achieved in less than $10 \%$ of patients with this clinical entity. The capsule endoscopy is considered an excellent non-invasive diagnostic method for the evaluation of the small bowel in this group of patients. All patients with melanoma history and gastrointestinal tract hemorrhage, iron deficiency anemia, abdominal pain and / or unexplained weight loss should perform an capsule endoscopy to rule out small bowel involvement. We present two patients with cutaneous melanoma with apparent remission who develop iron-deficiency anemia, without a history of evident gastrointestinal bleeding with negative endoscopic examinations and capsule endoscopy findings of intestinal metastasis due to melanoma.

Keywords. Capsule endoscopy, malignant melanoma, small bowel, iron deficiency anemia.

\section{Introducción}

El melanoma cutáneo es una neoplasia común y de comportamiento sumamente agresivo. Constituye 
$1-3 \%$ de todas las neoplasias y puede producir metástasis a diversos órganos, ya sea por vía hematógena o linfática, siendo los principales lugares de afectación el tracto gastrointestinal y el sistema nervioso central. Además se sabe que el $50 \%$ de los pacientes que tienen compromiso del tracto gastrointestinal por melanoma maligno ya tienen compromiso de otros órganos. ${ }^{1,2}$

La ubicación más frecuente de las metástasis, a nivel del tracto digestivo, es el intestino delgado, con predilección por la zona yeyuno-ileal. Seguida por la afectación gástrica, en un $30 \%$ de los casos, y con una prevalencia menor a nivel colónico y esofágico. El tipo histológico que genera metástasis con mayor frecuencia a intestino delgado es el superficial, aunque cualquier tipo de melanoma cutáneo podría hacerlo. ${ }^{3}$

Así mismo el melanoma metastásico intestinal tiene 4 formas de presentación: polipoidea, cavitaria, infiltrante y exoentérica, siendo la forma polipoidea la que con mayor frecuencia genera intususcepción intestinal. Sin embargo, la gran mayoría de pacientes son asintomáticos o presentan un cuadro clínico inespecífico que conlleva a un diagnóstico tardío y a un mal pronóstico a mediano-largo plazo..$^{4,5}$

Presentamos dos casos con anemia ferropénica, asociada a síntomas gastrointestinales inespecíficos, sin pérdidas sanguíneas evidentes, con endoscopías alta y baja normales, y en cuyo estudio de cápsula endoscópica se logró identificar datos compatibles con melanoma mestastásico intestinal, confirmados por vía quirúrgica.

\section{Casos clínicos}

\section{Caso No 1}

Paciente varón de 73 años, con un tiempo de enfermedad de 5 meses, caracterizado por presentar descenso de la hemoglobina de 13,7 a 11,8 g/dl, que no mejoró con la administración de hierro oral, sin historia de pérdidas gastrointestinales manifiestas. El paciente presentaba el antecedente de que 14 años antes había sido operado de un melanoma en el brazo derecho, así mismo 4 años antes se le había extraído un ganglio de la región axilar que fue informado como melanoma, para el cual recibió tratamiento, y cuyos controles anuales posteriores fueron negativos. Al examen clínico se evidenció palidez en piel y mucosas, así como también las cicatrices previas a nivel axilar y en brazo derecho; el resto del examen físico fue normal. Se realizaron endoscopía digestiva alta y colonoscopía completa, que fueron normales. El laboratorio confirmó niveles de ferritina y saturación de transferrina por debajo de lo normal. Se realizó una cápsula endoscópica, que mostró, aproximadamente a nivel de yeyuno, una lesión de aspecto proliferativo, parcialmente estenosante, ulcerada y friable con escaso sangrado activo (Figura $1 \mathrm{~A}-\mathrm{D})$. Con la sospecha de neoplasia de intestino delgado, se decidió realizar una enterotomografía que reveló un engrosamiento de aspecto tumoral a nivel de las paredes de yeyuno proximal presentando un área de 53 x $38 \mathrm{~mm}$ de ancho, paredes con un espesor de $14-17 \mathrm{~mm}$ y con una pequeña luz en medio de la masa parietal yeyunal (Figura 2 A y B).

El paciente fue sometido a laparotomía exploratoria, en la que se encontró una obstrucción intestinal por múltiples tumoraciones de aspecto metastásico asociado a intususcepción en dos puntos por fuera de la zona de metástasis (Figura 3).

Finalmente el estudio anatomopatológico reveló una neoformación sólida ulcerada de 7 × 5 x $2,5 \mathrm{~cm}$ de color pardo con hallazgos microscópicos compatibles con melanoma. El estudio de inmunohistoquímica mostró S100 y Melan A positivos, lo que concluyó como lesiones metastásicas de origen melanótico ( $\mathrm{Fi}$ gura 4 A y B).

\section{Figura 1. A y B) Lesión proliferativa parcialmente estenosante.}

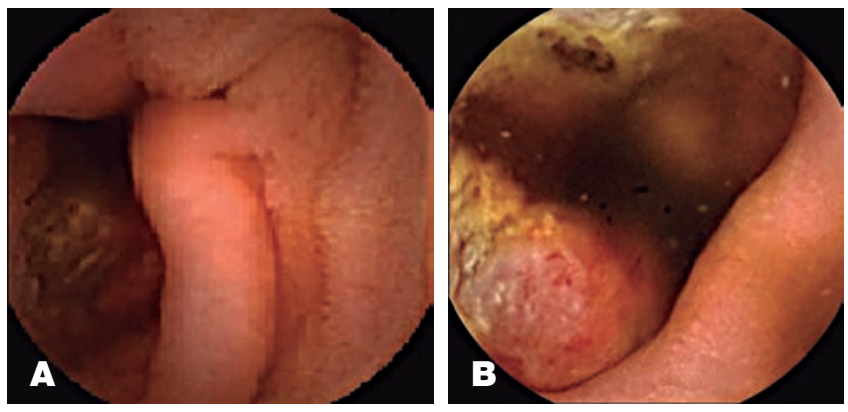

Figura 1. Cy D) Presencia de ulceraciones con escaso sangrado activo en napa.
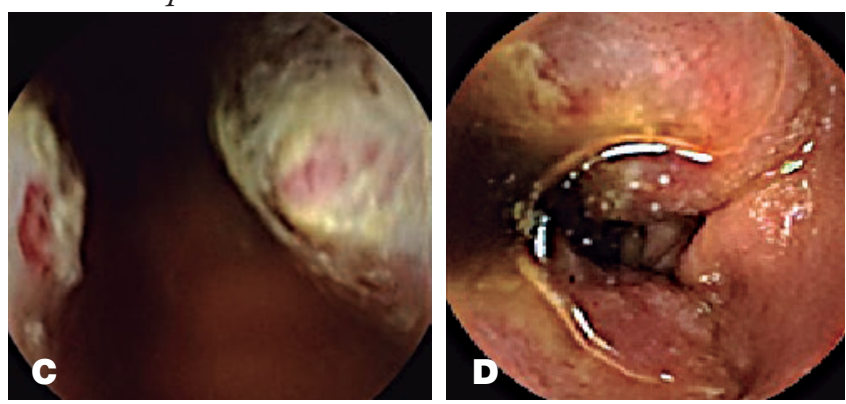
Tabla 2. A) Engrosamiento de aspecto tumoral a nivel de las paredes de yeyuno proximal. B) Presencia de pequeña luz en medio de la masa parietal yeyunal.
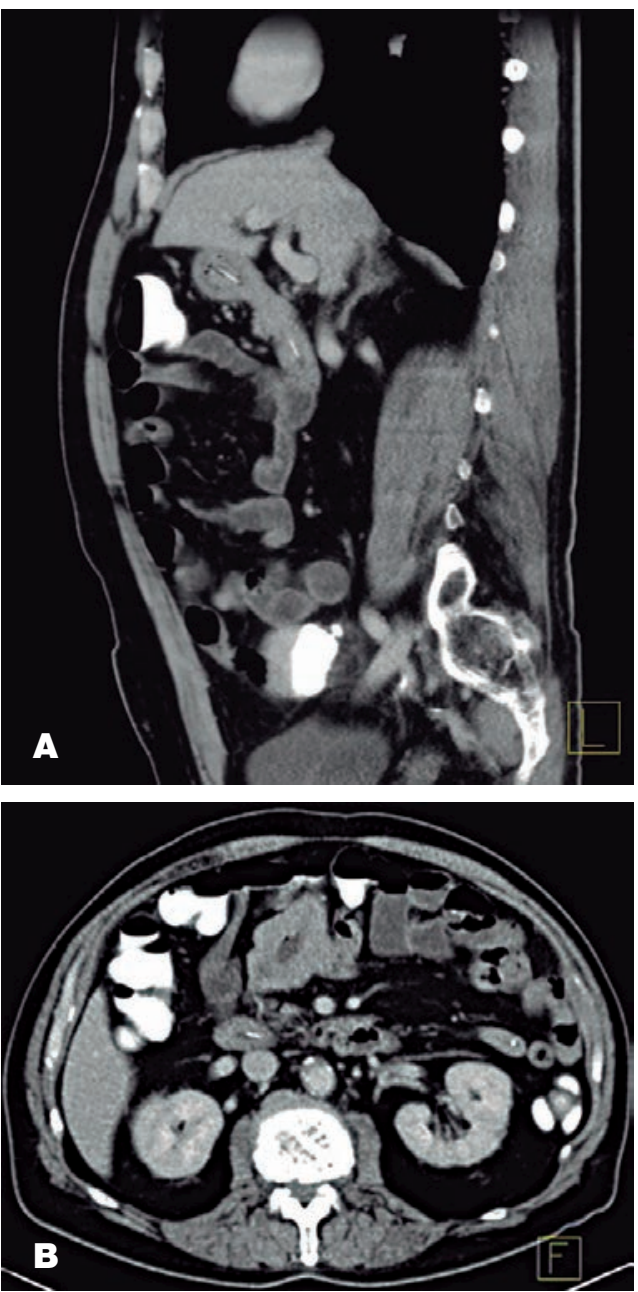

Figura 3. Múltiples tumoraciones de aspecto metastásico asociado a intususcepción en dos puntos por fuera del áreas de metástasis.

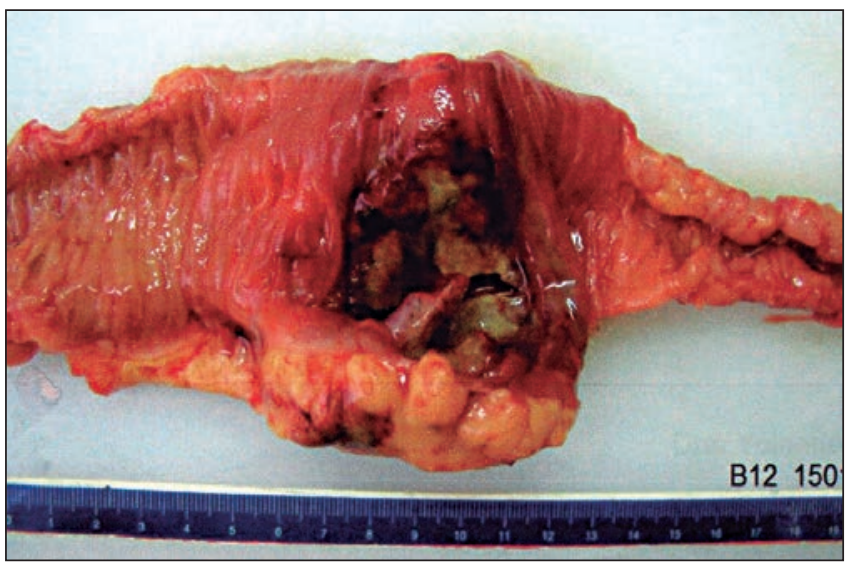

Figura 4. A) Tinción H-E (40X): infiltrado intestinal por células atípicas de aspecto melanocitico. B) Tinción Melan A: positivo para melanoma maligno.
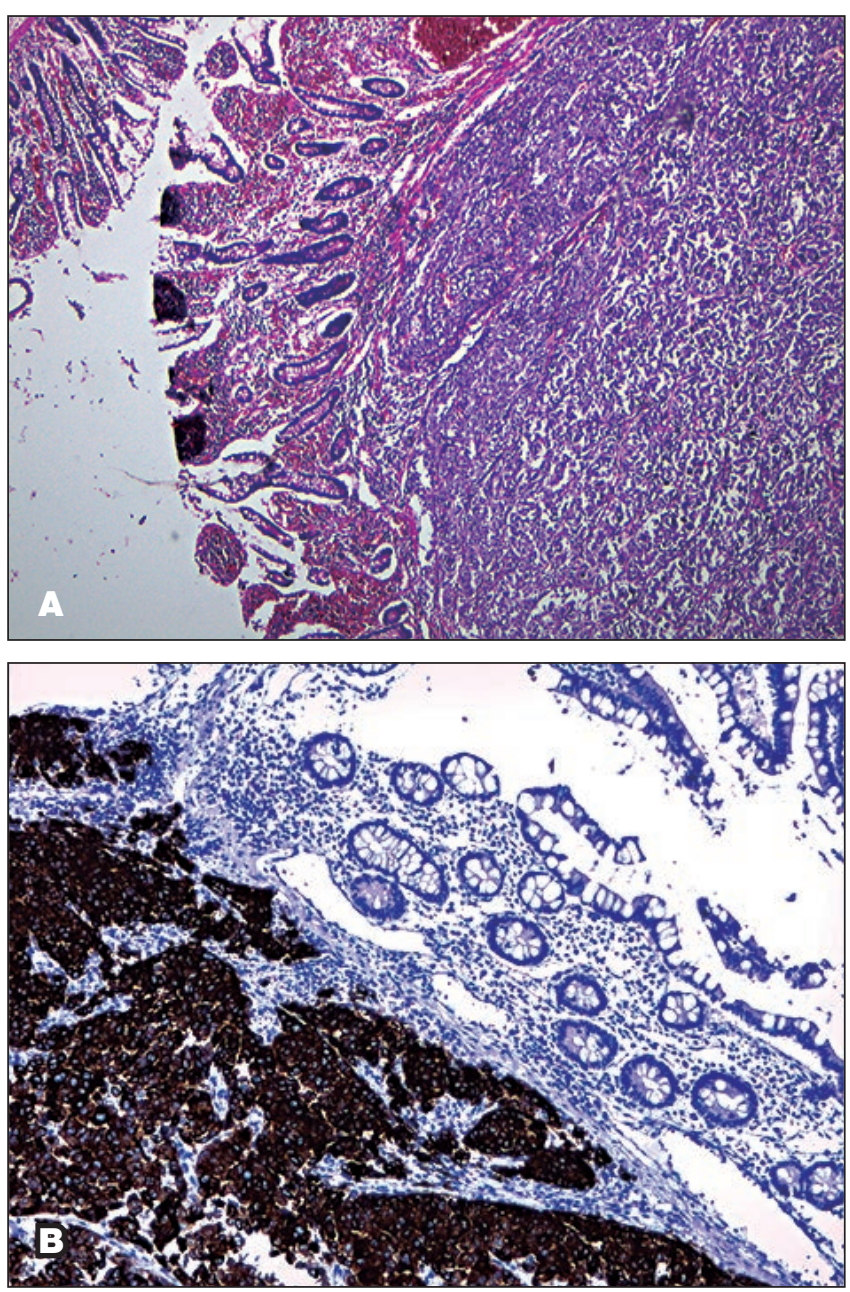

\section{Caso No 2}

Paciente varón de 67 años con tiempo de enfermedad de 2 meses, con dolor abdominal esporádico, además de debilidad y astenia asociado a náuseas, vómitos, dolor abdominal intenso y distensión abdominal. Hemoglobina de 7,9 gr/dl. Como antecedente de importancia se le había realizado hacía 3 años una resección de melanoma ubicado en la región facial izquierda asociado a tratamiento coadyuvante con quimio y radioterapia. Un mes antes del ingreso había sido hospitalizado por anemia severa sin pérdidas gastrointestinales manifiestas con requerimiento transfusional. Se le realizó endoscopía digestiva alta y colonoscopía, ambas normales. Al examen clínico se evidenció palidez en piel y mucosas, abdomen distendido, ruidos hidroaéreos disminuidos y dolor a la palpación profunda en mesogastrio. Se realizó 
una cápsula endoscópica que mostró aproximadamente entre duodeno distal y yeyuno múltiples lesiones elevadas, algunas de ellas ulceradas con sangrado en napa, de aspecto proliferativo que, considerando el antecedente previo, fueron sugestivas de lesiones metastásicas a nivel de intestino delgado. (Figura 5 A-D). Posteriormente se le realizó un estudio de enterotomografía donde se evidenció engrosamiento difuso e irregular de la pared intestinal aproximadamente a nivel yeyunal, las cuales fueron informadas como lesiones de aspecto metastásico infiltrantes (Figura 6 A y B).

Durante su hospitalización cursó con mayor distensión abdominal y persistencia de vómitos por lo que fue sometido a laparotomía exploratoria en donde se encontraron múltiples tumoraciones hiperpigmentadas y de consistencia pétrea de aproximadamente $5 \mathrm{~cm}$ de diámetro dependientes de yeyuno (Figura 7).

El estudio anatomopatológico reveló un segmento de $74 \times 10 \mathrm{~cm}$, con presencia de 15 tumoraciones de color pardo negruzco que medían entre 1,3 a $6 \mathrm{~cm}$ de diámetro con hallazgos compatibles con melanoma maligno que comprometía la pared intestinal de forma multifocal con bordes quirúrgicos libres de neoplasia (Figura 8 A y B).

Figura 5. $\boldsymbol{A}$ y B) Lesiones elevadas hiperpigmentadas de aspecto proliferativo. $\boldsymbol{C} \boldsymbol{y} \boldsymbol{D})$ Presencia de ulceraciones con escaso sangrado activo en napa.
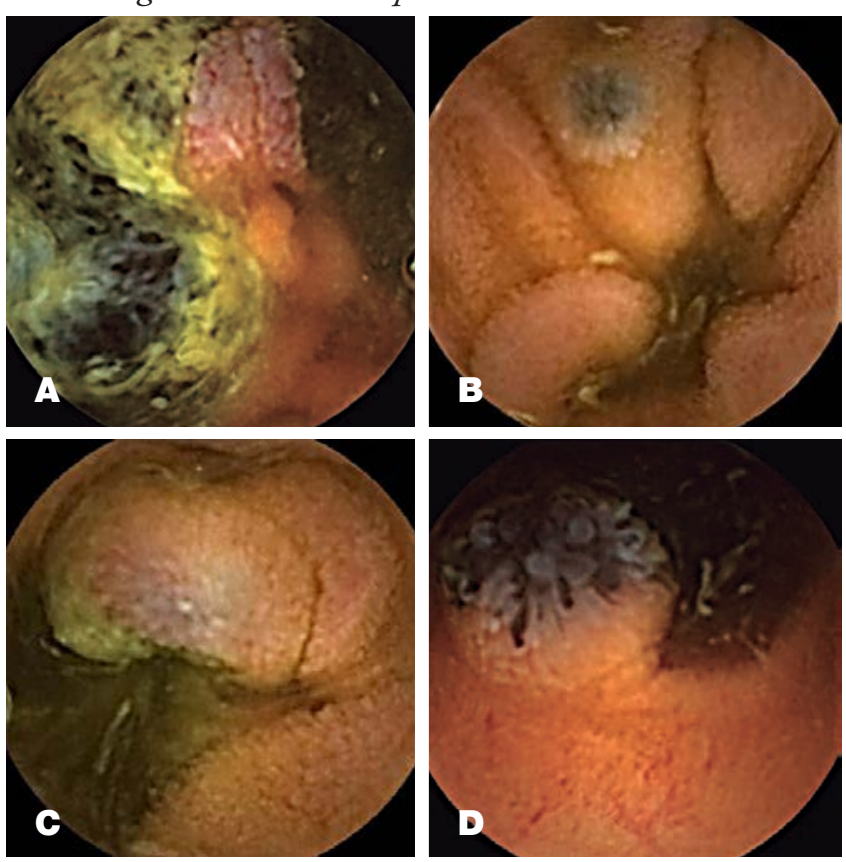

Figura 6. A) Engrosamiento difuso e irregular de paredes yeyunales de aspecto infiltrativo. B) Estrechamiento de luz yeyunal circundante a la lesión proliferativa.
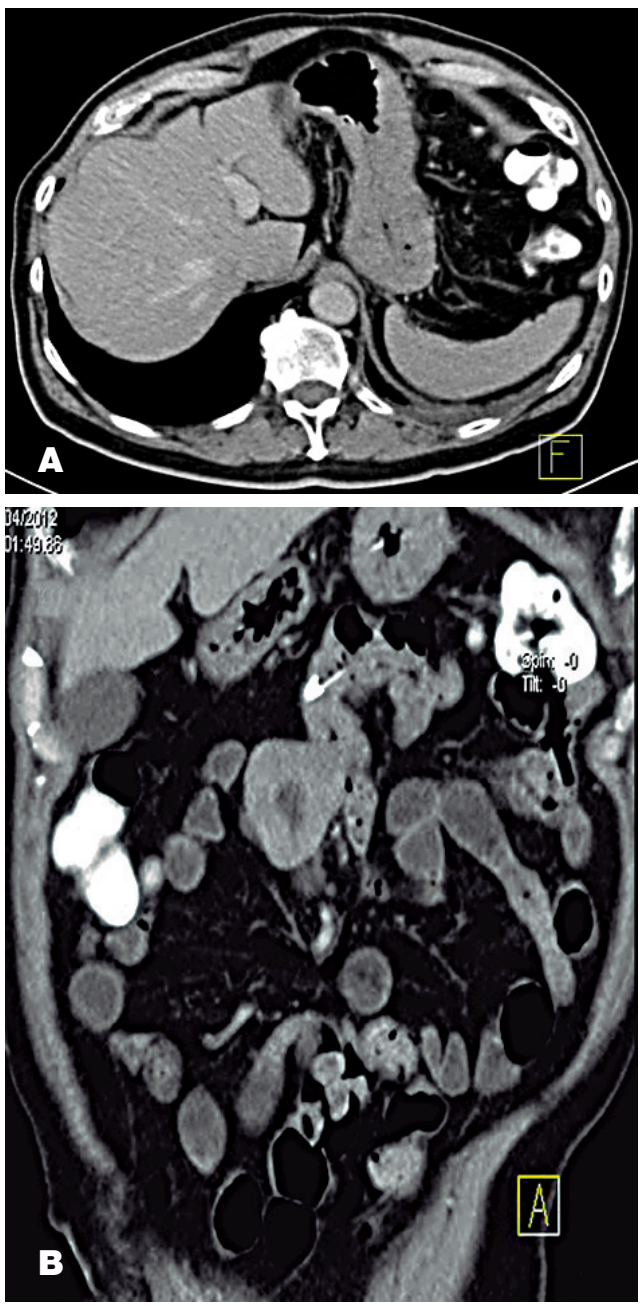

Figura 7. Múltiples tumoraciones hiperpigmentadas de consistencia pétrea a nivel de yeyuno.

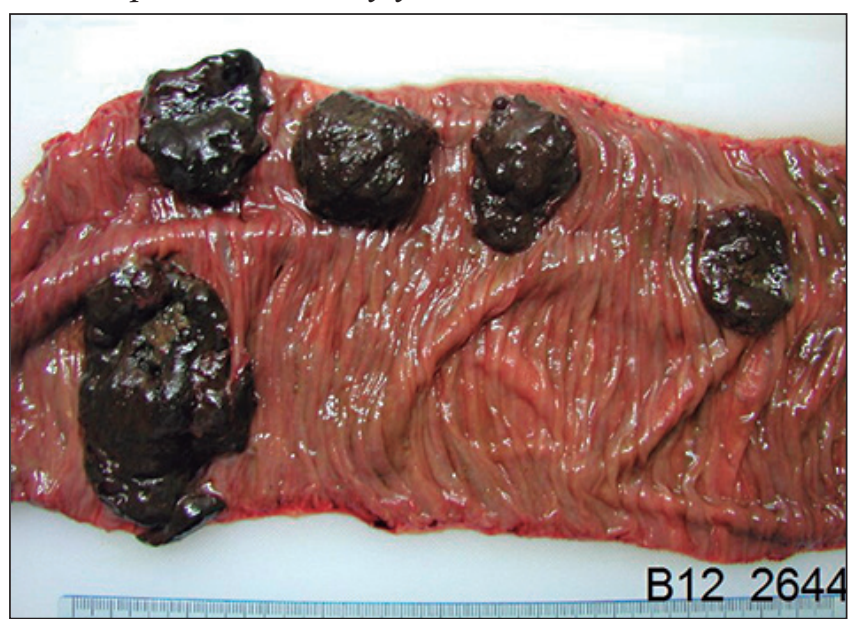


Figura 8. A) Tinción H-E (40X): iInfiltración intestinal por células atípicas de aspecto melanotico. B) Tinción especial S-100 positivo para melanoma maligno.
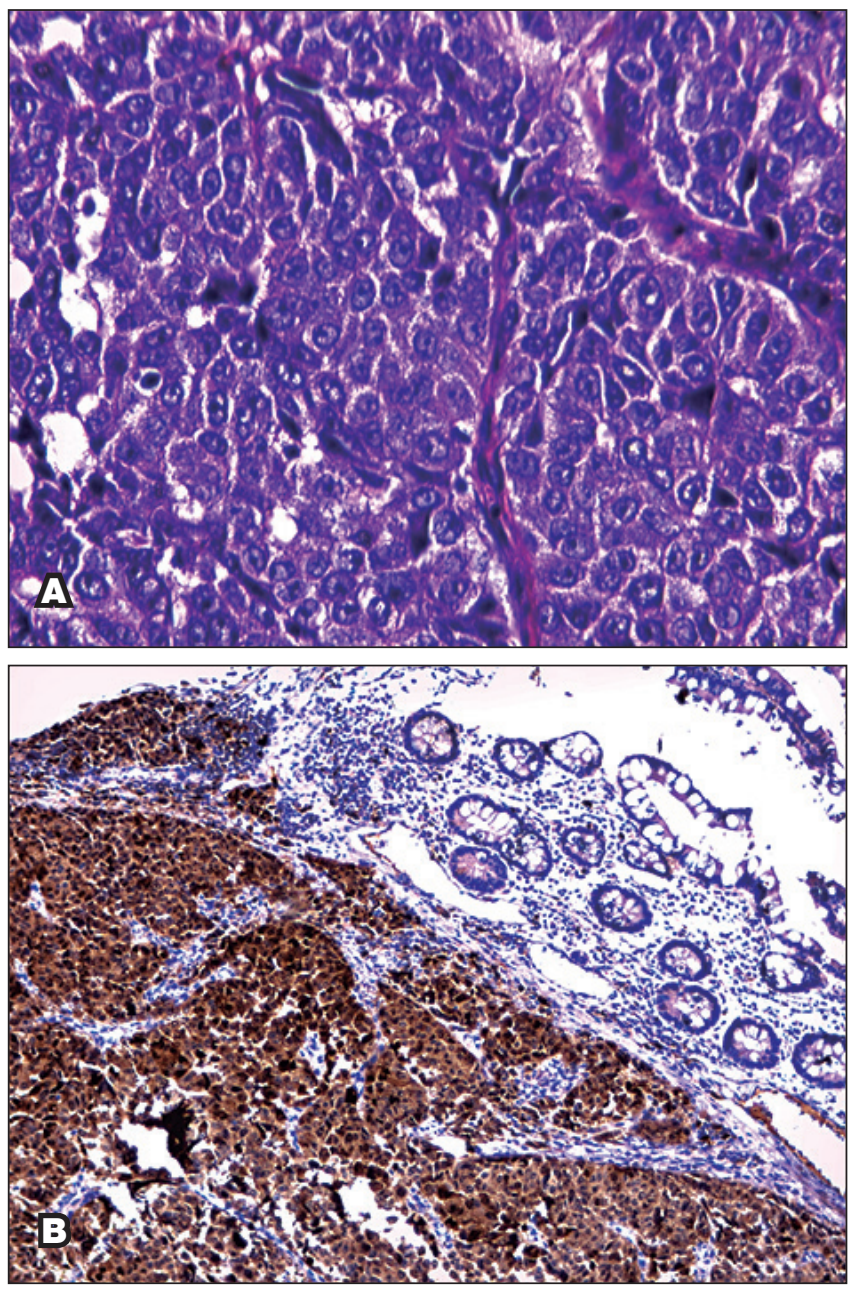

\section{Discusión}

El intervalo entre el diagnóstico del melanoma maligno primario y la detección de enfermedad metastásica es variable, entre 2 a 180 meses. ${ }^{1}$ El tiempo promedio, según las últimas series, entre el diagnóstico de melanoma maligno cutáneo y el desarrollo de metástasis a intestino delgado es de aproximadamente 3,2 años. ${ }^{2}$

Algunos de estos compromisos gastrointestinales han sido diagnosticados con técnicas endoscópicas convencionales, como el realizado por Bercholc-Urinowsky y col. ${ }^{3}$ en México, donde se presenta a un paciente varón de 67 años con antecedente de escisión local amplia de un melanoma ulcerado invasivo en espalda (Clark III- Breslow 1,8 mm) hacía 2 años, que debutó con hemorragia digestiva baja indolora con colonoscopía normal y endoscopía digestiva alta que reveló múltiples lesiones melanó- ticas ulceradas de diferente tamaño con histología compatible con metástasis gástrica por melanoma cutáneo. Antecedente de importancia que también presentaron nuestros pacientes.

Cabe resaltar que la presencia de melanomas intestinales no solo tiene un origen extra-gastrointestinal, ya que se han reportado casos de melanomas intestinales primarios. Mc.Quade y col. ${ }^{4}$ en Irlanda mostraron al realizar una colonoscopía a un paciente varón de 85 años, sin antecedentes de importancia, con historia de baja ponderal marcada asociado a trastornos en el hábito defecatorio, una lesión hiperpigmentada en cara anterior de la unión recto-sigmoidea con histopatología compatible con melanoma maligno, con lo que se concluyó como primario. Esto confirma que la localización más frecuente de estas lesiones melanóticas malignas primarias son a nivel anorectal y esófago proximal.

Diferentes grupos de investigación han diagnosticado casos de melanomas metastásicos intestinales inaccesibles a las técnicas endoscópicas convencionales por imágenes, como el estudio contrastado baritado, la tomografía abdominal computarizada, enterotomografía o enterorresonancia magnética nuclear, ${ }^{5}$ cuando por lo general el paciente presenta alguna manifestación clínica evidente, siendo confirmados por vía quirúrgica. ${ }^{6}$

Las series que hacen referencia a las manifestaciones clínicas del melanoma maligno metastásico en intestino delgado son escasas. En el estudio de Bender de 32 pacientes con melanoma maligno, $18(60 \%)$ presentaron dolor abdominal como síntoma predominante, 15 (47\%) obstrucción intestinal, 13 (41\%) náuseas y/o vómitos, $30 \%$ sangrado gastrointestinal y solo 3 (10\%) masa abdominal palpable. ${ }^{7}$ Cabe resaltar que en este estudio la anemia ferropénica no fue un hallazgo estadísticamente significativo, sin embargo fue el problema cardinal en nuestros pacientes.

La tomografía abdominal computarizada tiene una sensibilidad diagnóstica entre 50\%-70\% para la detección de este tipo de lesiones metastásicas, pero esta ha sido superada por la tomografía con emisión de positrones, por lo que algunos estudios la proponen como una técnica adecuada para la detección temprana de melanomas intestinales metastásicos. ${ }^{8}$ Sin embargo, actualmente múltiples trabajos de investigación han demostrado que el estudio por cápsula endoscópica es una herramienta fundamental en la evaluación inicial de intestino medio para diversas patologías a este nivel, entre ellas las tumoraciones de intestino delgado, como son los melanomas metastásicos intestinales. ${ }^{9} 10$ Tal y como se demostró en el estudio publicado este año por Flemming y col., ${ }^{11}$ en el cual de 203 pacientes sometidos a estudio de cápsula 
endoscópica por diversas indicaciones, entre ellas las más frecuentes hemorragia digestiva media y anemia ferropénica, se evidenció lesión en $85 \%$ de ellos (173 pacientes), siendo el hallazgo más común la presencia de erosiones y ulceraciones $(75 \%)$ seguidos de alteraciones vasculares tipo angiodisplásias (43\%). Así mismo se observó que del total de pacientes a los que se les realizó este procedimiento endoscópico, se tomaron medidas terapéuticas en $73 \%$ (149) y se modificó el tratamiento instaurado en $66 \%$ (133), lo cual demuestra el rol trascendental de la cápsula endoscópica en la evaluación del intestino delgado, que logra mejoras diagnóstico-terapéuticas en la mayoría de los casos. Se sabe además que la frecuencia de tumores de intestino delgado desde la aparición de la cápsula endoscópica fluctúa entre 2,4\%-9,6\% de los casos examinados. ${ }^{12}$ Uno de los estudios más representativos sobre este punto fue el elaborado por Prakoso y col. ${ }^{13}$ en Australia, en el que encuentran que el rendimiento diagnóstico para la detección de melanomas intestinales metastásicos por cápsula endoscópica es superior al generado por la tomografía con emisión de positrones, concluyendo que este examen endoscópico es una opción atractiva tanto para la investigación como para el seguimiento cercano e inicio de programas de detección temprana en pacientes de alto riesgo de metástasis a intestino medio por melanoma maligno. Sin embargo, algunas publicaciones actuales sugieren el uso complementario de ambas técnicas para un diagnóstico más certero. ${ }^{14}$

Uno de los parámetros más importantes que incrementan el riesgo de desarrollo de metástasis intestinal por melanoma maligno cutáneo es el nivel de invasión tumoral en profundidad expresado por el estadio clínico. Es con base en este criterio y la necesidad de identificación temprana de estas lesiones metastásicas en pacientes de alto riesgo que J. Albert y col. ${ }^{15}$ elaboraron un algoritmo diagnóstico a partir de un estudio multicéntrico, en donde se demostró que todo paciente con un melanoma maligno cutáneo en estadio clínico III y IV (según la American Joint Committee on Cancer) debe ser sometido inmediatamente después del diagnóstico a un seguimiento cercano en búsqueda de compromiso intestinal por este tipo de metástasis, poniendo énfasis en la gran utilidad de la cápsula endoscópica para este fin, incluso en pacientes que fueron sometidos a resección quirúrgica o remisión espontánea del foco primario dérmico, ya que se ha observado que aun con esta condición, este grupo de pacientes son susceptibles a la aparición de estas lesiones metastásicas intestinales en un tiempo promedio de a 4 a 6 años desde la resolución. ${ }^{16,17}$ Tal y como se presentó en cada uno de los 2 pacientes de nuestro reporte.
El impacto clínico de la detección temprana de este tipo de lesiones metastásicas intestinales es la posibilidad de una resección quirúrgica que mejore el pronóstico y la supervivencia a corto y mediano plazo, ya que en estadios avanzados la cirugía no ha demostrado ningún tipo de beneficio estadísticamente significativo en estos pacientes. Además se sabe que la sola afectación visceral por melanoma maligno cutáneo acorta la sobrevida a los 5 años a menos del $10 \% .^{18}$

La investigación óptima para la detección de metástasis de intestino delgado en pacientes con melanoma aún no se ha determinado. Sin embargo, los resultados obtenidos en diferentes estudios sugieren que la cápsula endoscópica es el método ideal para hacerlo. A pesar de esto se necesitan estudios a mayor escala y con cohortes prospectivas que propongan un mayor tiempo de seguimiento para extrapolar esta conducta diagnóstico-terapéutica en todo paciente que presente o que tenga el antecedente de melanoma maligno, resaltando la importancia de la utilización de la cápsula endoscópica como método diagnóstico de primera línea para la detección temprana de este tipo de lesiones en intestino medio, para generar oportunidades terapéuticas que mejoren el pronóstico y la sobrevida en estos pacientes.

Agradecimientos. Se agradece a todo el servicio de Gastroenterologia de la Clinica Anglo-Americana por la amplia colaboracion en la realizacion del presente estudio.

Conflicto de intereses. Los autores declaran que en el presente trabajo de investigacion no se presentaron conflictos de intereses.

Sostén financiero. El presente estudio no contó con ningún aporte financiero por parte de ninguna institución en particular.

\section{Aviso de derechos de autor}

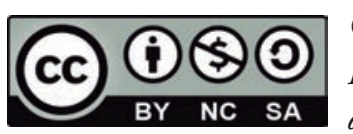

(C) 2021 Acta Gastroenterológica Latinoamericana. Este es un artículo de acceso abierto publicado bajo los términos de la Licencia Creative Commons Attribution (CC BY-NC-SA 4.0), la cual permite el uso, la distribución y la reproducción de forma no comercial, siempre que se cite al autor y la fuente original.

Cite este artículo como: Cedron Cheng H, Aliaga Ramos J. Cápsula endoscópica para el diagnóstico de metástasis de melanoma en intestino delgado, reporte de dos casos y revisión de la literatura. Acta Gastroenterol Latinoam. 2020;50(1):50-6. https://doi.org/10.52787/llbj9802 


\section{Referencias}

1. Lens M, Bataille V, Krivokapic Z. Melanoma of the small intestine. Lancet Oncol 2009; 10 (1): 516-521.

2. Liang K, Sanderson S, Nowakowski G, et al. Metastatic malignant melanoma of the gastrointestinal tract. Mayo Clin Proc 2006; 81 (4): 511-516.

3. Bercholc-Urinowsky I, Aristi-Urista G, Shueke-Esses M. Diagnóstico endoscópico incidental de metástasis gástrica por melanoma cutáneo. Revista de Gastroenterología de México 2017; 82 (4): 337-338.

4. Mc Quade C, Peadar S, O'Brien C, et al. Colorectal intussusception secondary to primary rectal melanoma: A novel case report. International Journal of Surgery Case Reports 2018; 44 (2): 78-81.

5. Giakoustidis A, Goulopoulos T, Boutis A y col. Jejunojejunal Intussusception due to Metastatic Melanoma Seven Years after the Primer: Case Reports in Surgery 2017; 3 (1): 131-135.

6. Franco-Herrera R, Burneo-Esteves M, Fabregues-Olea A, et al. Invaginación intestinal en el adulto. Una causa infrecuente de obstrucción mecánica. Revista de Gastroenterología de México 2012; 77 (3): 153-156.

7. Bender G, Maglinte D, Mc. Larney J, et al. Malignant melanoma: Patterns of metastasis to the small bowel, reliability of imaging studies, and clinical relevance. Am J Gastroenterol 2001; 96 (2): 2392-2400.

8. Swetter S, Carroll L, Johnson D, et al. Positron emission tomography is superior to computed tomography for metastatic detection in melanoma patients. Ann Surg Oncol 2002; 9 (2): 646-653.
9. Bailey A, Debinski H, Appleyard M, et al. Diagnosis and Outcome of Small Bowel Tumors Found by Capsule Endoscopy: A Three-Center Australian Experience. American Journal of Gastroenterology 2006; 101 (2): 2237-2243.

10. Shyung L, Lin S, Shih S, et al. Proposed Scoring System to Determine Small Bowel Mass Lesions Using Capsule Endoscopy. J. Formos Med Assoc 2009; 108 (7): 533-538.

11. Flemming J, Cameron S. Small bowel capsule endoscopy. Indications, results and clinical benefit in a University environment. Journal Medicine 2018; 97 (14): 1-9.

12. Cedron H. Cápsula Endoscópica del Intestino Delgado. Rev Gastroenterol Perú 2010; 30 (4): 341-349.

13. Prakoso E, Fulham M, Thompson J, et al. Capsule endoscopy versus positrón emission tomography for detection of small bowel metastatic melanoma: a pilot study. Gastrointestinal Endoscopy 2011; 73 (4): 750-756.

14. Tatlidil R, Mandelkern M. FDG-PET in the detection of gastrointestinal metastases in melanoma. Melanoma Res 2001; 11 (2): 297-301.

15. Albert J, Fechner M, Fiedler E, et al. Algoritmo for detection of small-bowel metastasis in malignant melanoma of the skin. Endoscopy 2011; 43 (3): 490-498.

16. Prakoso E, Selby W. Review: Capsule Endoscopy for Management of Small Bowel Melanoma Is It Time Yet? Gastroenterology \& Hepatology 2012; 10 (8): 694-695.

17. Issa H, Poovathumkadavil A, Almousa F, et al. Metastatic Malignant Melanoma of the Small Intestines Diagnosed by Capsule Endoscopy. Journal compilation Gastroenterology Research: Case Report 2011; 4 (5): 236-239.

18. Hodgson R, Fink M, Jones R. The role of abdominal resectional surgery in metastatic melanoma. Journal compilation 2007; 77 (2): $855-859$. 